\title{
An International Urogynecological Association (IUGA)/International Continence Society (ICS) joint report on the terminology for female pelvic floor dysfunction
}

Haylen BT, de Ridder D, Freeman RM, Swift SE, Berghmans B, Lee J, Monga A, Petri E, Rizk DE, Sand PK, Schaer GN

University of New South Wales, Sydney, New South Wales, Australia

Neurourol Urodyn. 2010;29(1):4-20.

Introduction: Next to existing terminology of the lower urinary tract, due to its increasing complexity, the terminology for pelvic floor dysfunction in women may be better updated by a female-specific approach and clinically based consensus report.

Methods: This report combines the input of members of the Standardization and Terminology Committees of two international organizations, the International Urogynecological Association (IUGA), and the International Continence Society (ICS), assisted at intervals by many external referees. Appropriate core clinical categories and a subclassification were developed to give an alphanumeric coding to each definition. An extensive process of 15 rounds of internal and external review was developed to exhaustively examine each definition, with decision-making by collective opinion (consensus).

Results: A terminology report for female pelvic floor dysfunction, encompassing over 250 separate definitions, has been developed. It is clinically based with the six most common diagnoses defined. Clarity and user-friendliness have been key aims to make it interpretable by practitioners and trainees in all the different specialty groups involved in female pelvic floor dysfunction. Female-specific imaging (ultrasound, radiology, and MRI) has been a major addition while appropriate figures have been included to supplement and help clarify the text. Ongoing review is not only anticipated but will be required to keep the document updated and as widely acceptable as possible.

Conclusion: A consensus-based terminology report for female pelvic floor dysfunction has been produced aimed at being a significant aid to clinical practice and a stimulus for research.

\section{Editorial Comment}

This is a very noteworthy review article which should be kept as a reference point for the various terminologies and definitions used in the contemporary literature. It may hold a keen value when preparing manuscripts for publication. As stated in the article, this terminology report is user friendly, clinically based, and quite explanatory in its description. That it was developed by leaders of the specialties concerned with pelvic floor dysfunction, including what appears to be an exhaustive number of internal and external reviews and evaluations, lends to its' value and strength as a reference article. Also to the interested party, reading the entire journal in which this article is published (Neurourology and Urodynamics, Vol. 29(1), 2010) is of good intellectual value with regards to the time expended and subsequent knowledge gleaned.

\author{
Dr. Steven P. Petrou \\ Professor of Urology, Associate Dean \\ Mayo School of Graduate Medical Education \\ Jacksonville, Florida, USA \\ E-mail: petrou.steven@mayo.edu
}

